\section{Degree of Vasculopathy in Systemic Sclerosis Patients with Anti-U3RNP Antibody Indicates Need for Extensive Cardiopulmonary Screening}

\section{To the Editor:}

In patients with systemic sclerosis (SSc), regular screening is needed to determine the extent and severity of organ involvement ${ }^{1}$. Specific autoantibodies are associated with clinical manifestations and are therefore used as predictors for organ involvement ${ }^{2}$. Identifying patients who are at risk for organ involvement enables distinguishing between patients with high risk who need extensive screening and followup, and patients with most likely mild disease. Antibodies against the U3RNP (or antifibrillarin) are detected in 3-8\% of patients with SSc. Some studies indicate a higher risk for cardiac involvement in U3RNP+ patients, but results are conflicting $3,4,5,6,7$. As shown before, the degree of microangiopathy as reflected by nailfold videocapillaroscopy (NVC) is identified as an independent predictor for organ involvement in $\mathrm{SSc}^{8}$. Associations between presence of U3RNP and NVC pattern and disease manifestations have not been evaluated to date Therefore, we evaluated degree of microangiopathy as shown by NVC and its association with cardiopulmonary involvement in anti-U3RNP+ patients with SSc.

All patients participating in the Combined Care in Systemic Sclerosis (CCISS) prospective observational cohort study (approved by the Ethics Committee P09.003/SH/sh) of the Leiden University Medical Center gave written informed consent. Eighteen U3RNP+ patients (indicating a prevalence of $3 \%$ in the CCISS cohort) were compared with an equal number of age- and sex-matched anticentromere+ (ACA) and antitopoisomerase+ (ATA) controls. Although controls were not matched for disease duration, there were no significant differences between the groups. Cardiopulmonary screening was performed routinely in all patients.

Myocardial involvement was defined using a combined value in which patients had to have at least 2 of the following: arrhythmias (> $2 \%$ ventricular/supraventricular arrhythmia or atrial fibrillation), conduction problems (atrioventricular/bundle branch blocks), left ventricular ejection fraction $<50 \%$, diastolic dysfunction, or pericardial effusion. Pulmonary involvement was defined based on the combination of DLCO $<60 \%$ and forced vital capacity $<70 \%$ and evidence for interstitial lung disease on high-resolution computed tomography. NVC was performed at baseline, and images were classified by a trained observer as early, active, or late SSc pattern $^{9}$. In addition, capillary loss $(<7$ per $\mathrm{mm})$ was determined.
U3RNP+ patients were most often female (78\%), with a high prevalence of diffuse cutaneous SSc (39\%); $22 \%$ were of African American origin $(n=4)$ compared to $0 \%$ among the ACA+ and ATA+ patients. This confirms the results of other studies in which an association between U3RNP antibody, skin score, and African American race was found.

Myocardial involvement was present in $17 \%(\mathrm{n}=3)$ of the U3RNP+ patients compared to $17 \%(n=3)$ in the ACA+ and $33 \%(n=6)$ in the ATA+ patients. Pulmonary involvement was present in $6 \%(\mathrm{n}=1)$ of the U3RNP+ patients compared to $6 \%(n=1)$ in the ACA+ and $29 \%(n=5)$ in the ATA+ patients. Based on these data, we cannot confirm a higher prevalence of cardiopulmonary involvement in patients with U3RNP antibody compared with ACA+/ATA+ patients. Because mean skin scores and pulmonary function test results were clearly worse in U3RNP+ patients as compared to ACA patients, indicating more severe disease with possibly higher risk, we consequently evaluated whether degree of microangiopathy could help distinguish those U3RNP patients at risk for cardiopulmonary involvement. The distribution of SSc patterns among U3RNP+ and ATA+ patients was comparable; in the ACA+ patients a late SSc pattern and capillary loss were less common (Figure 1). For the complete population with NVC available $(n=45)$, late NVC pattern was associated with pulmonary involvement (chi squared, $\mathrm{p}=0.009$; Table 1 ) and capillary loss with myocardial involvement (chi squared, $\mathrm{p}=0.05$; Table 1 ). Nevertheless, it is the severity of microangiopathy that is associated with both cardiac and pulmonary involvement, and we do not consider late pattern and capillary loss as different pathophysiological processes. When selecting all U3RNP+ patients $(n=18)$ for extensive cardiopulmonary screening, cardiopulmonary involvement was detected in $11 \%$ ( 2 out of 18 ). When only selecting U3RNP+ patients with either late SSc pattern or capillary loss, extensive screening was necessary in $47 \%$ of U3RNP+ patients ( 7 out of 15 patients), and none of the U3RNP+ patients with cardiopulmonary involvement was missed.

To our knowledge, this is the first study to describe NVC findings in U3RNP+ patients with SSc, and to associate NVC patterns with cardiopulmonary involvement in this group. The strength of the presented data lies in the prospective study design with standardized annual followup independent of clinical presentation. However, given the low numbers, our findings need replication. As previously shown, U3RNP antibody was more prevalent among African Americans. We could not confirm previous suggestions of a higher prevalence of cardiac involvement in this group. Our data confirm that in SSc, the degree of microangiopathy as reflected by NVC is associated with severity of cardiopulmonary involvement. This is also true

\title{
NVC pattern
}

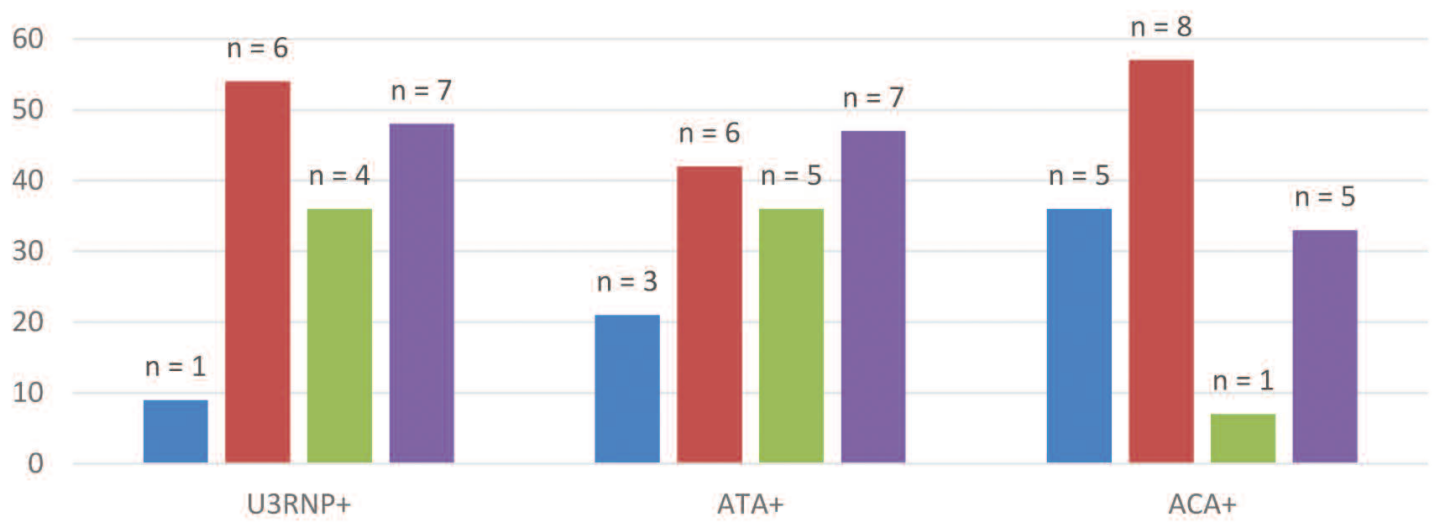

Early SSc pattern, $n(\%)$ active SSc pattern, $n(\%)$ - Late SSc pattern, $n(\%)$ a Capillary loss, $n(\%)$

Figure 1. Distribution of nailfold videocapillaroscopy (NVC) patterns among the autoantibodies. In the U3RNP group, 3 patients had secondary pattern and 1 an aspecific pattern. In the ACA and ATA groups, only 1 patient had a secondary pattern (in the ACA group). SSc: systemic sclerosis; ATA+: antitopoisomerase-positive patients; ACA+: anticentromere-positive patients.

Personal non-commercial use only. The Journal of Rheumatology Copyright @ 2019. All rights reserved. 
Table 1. Total group and U3RNP+ group, SSc pattern, capillary loss, and organ involvement.

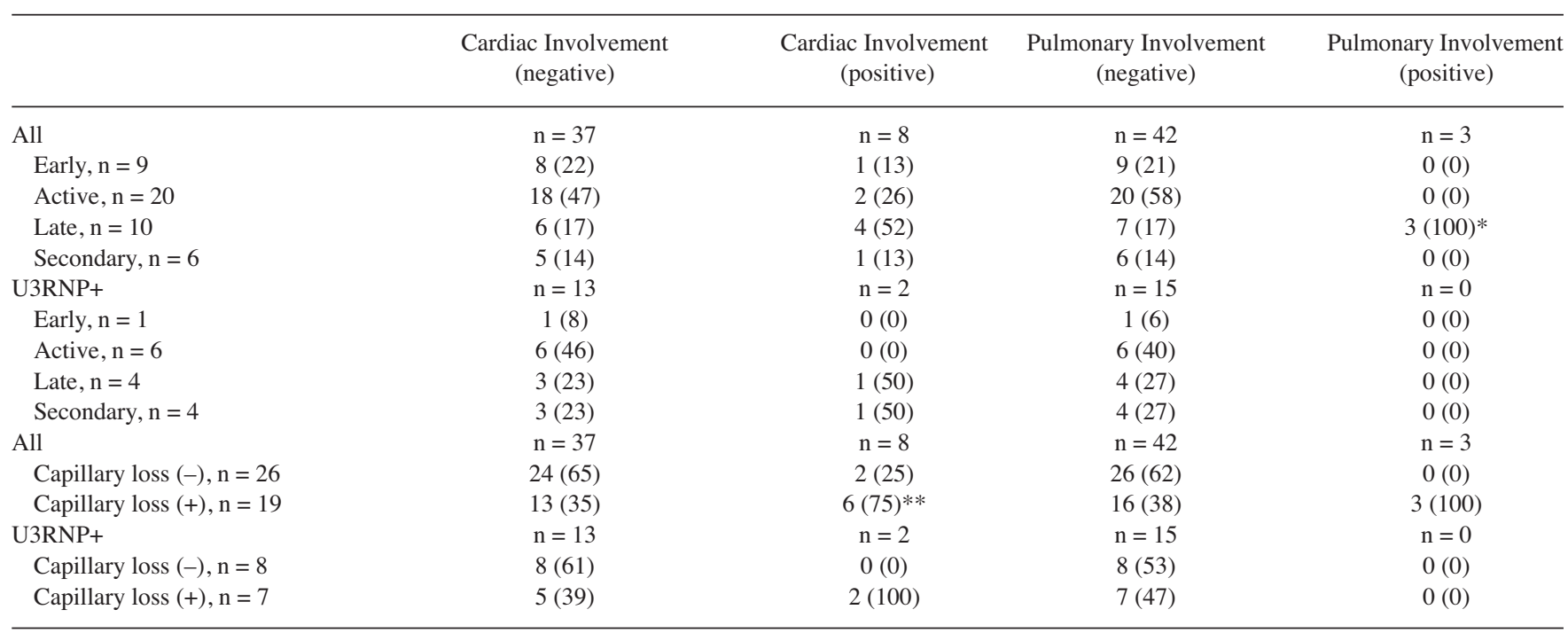

Values are $\mathrm{n}(\%)$ unless otherwise specified. $* \mathrm{P}$ value $=0.009$ chi square; late pattern vs non late pattern. $* * \mathrm{P}$ value $=0.047$ chi square; capillary loss vs no loss, all other comparisons nonsignificant. Cardiac involvement defined as a Medsger score of 1 or higher, or at least 2 of the following: decreased LVEF, arrhythmias, conduction abnormalities, diastolic dysfunction, or pericardial effusion. Pulmonary involvement defined as decreased FVC and DLCO and ILD on HRCT. SSc: systemic sclerosis; LVEF: left ventricular ejection fraction; FVC: forced vital capacity; ILD: interstitial lung disease; HRCT: high-resolution computed tomography.

in $\mathrm{U} 3 \mathrm{RNP}+$ patients. NVC can serve as a biomarker to select U3RNP+ SSc patients with higher risk for cardiopulmonary involvement.

NINA M. VAN LEEUWEN ${ }^{\circ}$, MD, Leiden University Medical Center, Department of Rheumatology; MAAIKE BOONSTRA, MD, Leiden University Medical Center, Department of Rheumatology; NINA AJMONE MARSAN, MD, Leiden University Medical Center, Department of Cardiology; MAARTEN K. NINABER, MD, Leiden University Medical Center, Department of Pulmonology; TOM W.J. HUIZINGA, MD, PhD, Leiden University Medical Center, Department of Rheumatology; JESKA K. DE VRIES-BOUWSTRA, MD, Leiden University Medical Center, Department of Rheumatology, Leiden, the Netherlands. Address correspondence to Dr. N.M. van Leeuwen, Department of Rheumatology, Leiden University Medical Center, C1-R, PO Box 9600, 2300 RC, Leiden, the Netherlands.E-mail: n.m.van_leeuwen@lumc.nl

\section{REFERENCES}

1. Nihtyanova SI, Tang EC, Coghlan JG, Wells AU, Black CM, Denton CP. Improved survival in systemic sclerosis is associated with better ascertainment of internal organ disease: a retrospective cohort study. QJM 2010;103:109-15.

2. Mierau R, Moinzadeh P, Riemekasten G, Melchers I, Meurer M, Reichenberger F, et al. Frequency of disease-associated and other nuclear autoantibodies in patients of the German network for systemic scleroderma: correlation with characteristic clinical features. Arthritis Res Ther 2011;13:R172.

3. Tormey VJ, Bunn CC, Denton CP, Black CM. Anti-fibrillarin antibodies in systemic sclerosis. Rheumatology 2001;40:1157-62.
4. Mejia Otero C, Assassi S, Hudson M, Mayes MD, Estrada-Y-Martin $\mathrm{R}$, Pedroza C, et al; Genetics versus Environment in Scleroderma Outcome Study. Antifibrillarin antibodies are associated with native North American ethnicity and poorer survival in systemic sclerosis. J Rheumatol 2017;44:799-805.

5. Sharif R, Fritzler MJ, Mayes MD, Gonzalez EB, McNearney TA, Draeger $\mathrm{H}$, et al. Anti-fibrillarin antibody in African American patients with systemic sclerosis: Immunogenetics, clinical features, and survival analysis. J Rheumatol 2011;38:1622-30.

6. Aggarwal R, Lucas M, Fertig N, Oddis CV, Medsger TA, Jr. Anti-U3 RNP autoantibodies in systemic sclerosis. Arthritis Rheum 2009;60:1112-8.

7. Arnett FC, Reveille JD, Goldstein R, Pollard KM, Leaird K, Smith EA, et al. Autoantibodies to fibrillarin in systemic sclerosis (scleroderma). An immunogenetic, serologic, and clinical analysis. Arthritis Rheum 1996;39:1151-60.

8. Smith V, Riccieri V, Pizzorni C, Decuman S, Deschepper E, Bonroy $\mathrm{C}$, et al. Nailfold capillaroscopy for prediction of novel future severe organ involvement in systemic sclerosis. J Rheumatol 2013;40:2023-8.

9. Cutolo M, Pizzorni C, Tuccio M, Burroni A, Craviotto C, Basso M, et al. Nailfold videocapillaroscopic patterns and serum autoantibodies in systemic sclerosis. Rheumatology 2004;43:719-26.

J Rheumatol First Release July 1 2019; 2019;46:9; doi: $10.3899 /$ jrheum. 181378 